\title{
Les budgets globaux cantonaux ne répondent pas aux attentes
}

\author{
Bruno Trezzini ${ }^{\mathrm{a}}$, Beatrix Meyer
}

a Bruno Trezzini, Dr phil., collaborateur scientifique, division Médecine et tarifs hospitaliers, FMH; ${ }^{b}$ cheffe de la division Médecine et tarifs hospitaliers, FMH

Certains cantons espèrent endiguer la croissance des coûts dans le secteur hospitalier stationnaire à l'aide de budgets globaux. Une étude sur mandat de l'Office fédéral de la santé publique montre cependant que dans les cantons avec des budgets globaux, la croissance des coûts dans l'assurance obligatoire des soins n'est pas inférieure à celle des cantons sans budgets globaux. Du point de vue de la FMH, les possibles effets indésirables des budgets globaux doivent encore être soumis à une analyse détaillée. Il s'agit de se focaliser sur les alternatives disponibles.

Avec le nouveau financement hospitalier, le législateur voulait encourager la concurrence et réduire la croissance des coûts. En même temps, l'art. 51 al. 1 LAMal laisse aux cantons le choix de fixer, en tant qu'instrument de gestion des finances, un montant global pour le financement des hôpitaux. Un petit groupe de cantons (notamment Genève, le Tessin et Vaud, temporairement aussi Neuchâtel) ont jusqu'ici fait usage de cet instrument. Mais les budgets globaux au niveau cantonal sont-ils compatibles avec les objectifs et principes du nouveau financement hospitalier (y compris les forfaits par cas SwissDRG)? Et permettent-ils effectivement de freiner la croissance des coûts? La politique s'intéresse aussi à ces questions, comme le montre par exemple une initiative parlementaire de 2014 [1]. Ces questions sont aussi pertinentes du fait que le Conseil fédéral [2] examine actuellement, sur la base des recommandations d'un rapport d'experts [3], un objectif de maîtrise des coûts de la santé au niveau fédéral. Une étude établie sur mandat de l'Office fédéral de la santé publique (OFSP) relative aux budgets globaux cantonaux dans le contexte du nouveau financement hospitalier est disponible depuis peu [4]. Préalablement à la publication de l'étude, la FMH a eu l'occasion de prendre position de façon informelle.

\section{Les budgets globaux sont-ils compatibles avec le nouveau financement hospitalier?}

L'étude parvient à la conclusion que le budget global est en principe compatible avec les objectifs poursuivis dans le cadre du nouveau financement hospitalier [5], mais rend attentif à certains champs de tension [6]. Il s'agit notamment des différents principes du nouveau financement hospitalier tels que le choix étendu de l'hôpital, le financement dual-fixe et l'égalité de traitement des hôpitaux publics et privés. En effet, le choix de l'hôpital serait restreint si un hôpital inscrit sur la liste n'acceptait plus de patients pour cause d'épuisement du budget global. Suivant les circonstances, il faudrait établir des listes d'attente [7]. Et pour ce qui concerne le financement dual-fixe, la part cantonale effective passe sous les $55 \%$ lorsque le budget global est dépassé. A ce sujet, les auteurs se réfèrent à la jurisprudence du Tribunal fédéral selon laquelle cela est admis [8]. Concernant l'égalité de traitement entre les hôpitaux publics et privés dans l'application du budget global, ils estiment que celle-ci est respectée dans le canton du Tessin, contrairement aux cantons de Vaud et Genève, durant l'année sous étude (2017) [9]. Dans ce contexte, ils se réfèrent aussi à un récent arrêt du Tribunal administratif fédéral critiquant l'inégalité de traitement entre les hôpitaux publics et privés lors de l'attribution des budgets globaux et des volumes de prestations dans le canton de Genève [10].

Un autre point important du nouveau financement hospitalier est la rémunération liée aux prestations. Les auteurs admettent que le concept du budget global contredit d'une certaine façon le concept du financement des prestations, mais que la forme mixte utilisée par les cantons concernés en 2017 peut être considérée comme juridiquement compatible avec le nouveau financement hospitalier [11]. D’après les auteurs, c'est notamment le cas lorsque le calcul du budget global s'effectue sur la base des volumes de prestations (et non pas, p. ex., sur la base des coûts des années 
précédentes) [12]. Cela peut cependant être considéré sous différents angles: même si un budget global s'appuie sur les volumes de prestations prévisionnels ou passés, cela ne constitue pas, du moins d'un point de vue économique, une référence directe aux prestations. Il existe d'ailleurs d'autres doctrines juridiques et économiques sur la situation. Ainsi, le budget global est considéré comme étranger au modèle d'assurancemaladie existant [13] voire même comme un premier pas vers des caisses uniques cantonales [14].

\section{Croissance des dépenses comparable, aussi avec les budgets globaux}

Le tableau 1 résume les principaux résultats de l'analyse descriptive relative aux éventuels écarts entre les cantons avec et sans budgets globaux [15]. Il présente notamment les taux de croissance annuels dans le domaine AOS [16] pour la période 2012-2015. Les cantons avec budgets globaux enregistrent certes une croissance du volume plus faible que les cantons sans budgets globaux (0,6 vs. $1,2 \%)$, mais pour ce qui est des dépenses AOS hospitalières stationnaires dans les soins somatiques aigus, les taux de croissance sont les mêmes $(0,1 \%)$. Compte tenu des dépenses AOS totales, la croissance des cantons avec des budgets globaux n'est pas non plus inférieure $(2,7 \%)$. D'autres facteurs que le budget global sont également susceptibles d'influencer la croissance du volume et des coûts dans l'une ou l'autre direction. Par exemple, la croissance plus faible du volume des cantons avec des budgets globaux durant la période 2012-2015 dans le secteur des soins somatiques aigus stationnaires peut, du moins en partie, être attribuée à un effet de base qui favorise les cantons avec des budgets globaux.
En effet, les cantons sans budgets globaux avaient moins de cas AOS par tête dans le secteur des soins somatiques aigus stationnaires au début de la période d'analyse que ceux avec des budgets globaux [17]. Leur croissance du volume démarrait donc à un niveau inférieur à celui des cantons avec des budgets globaux, ce qui s'est traduit par un taux de croissance comparativement plus élevé. Sans cet effet de base, les cantons sans budgets globaux seraient donc en meilleure position. Si l'on tient aussi compte de l'évolution démographique, les coûts AOS dans le secteur des soins somatiques aigus stationnaires par tête augmentent moins fortement dans les cantons sans budgets globaux que dans les cantons avec budgets globaux [18]. En tenant compte de ces effets, les cantons sans budgets globaux ont obtenu de meilleurs résultats que ne le laisse penser le tableau 1. Il existe cependant aussi des effets contraires. Ainsi, l'ajustement progressif de la part can-

\section{Les cantons avec et ceux sans budgets globaux présentent une croissance des coûts AOS comparable durant la période d'analyse.}

tonale de financement à 55\% a contribué à un ralentissement de l'augmentation des dépenses AOS dans le groupe des cantons sans budgets globaux [19]. D'après les auteurs, la question de l'impact et de l'effet causal des budgets globaux sur les volumes de prestations et les coûts du domaine des soins somatiques aigus ne peut être tranchée de manière définitive sur la base de la présente étude [20].

Concernant les dépenses cantonales pour les prestations hospitalières stationnaires (y compris PIG [21] et investissements), le groupe des cantons avec budgets globaux présente une croissance plus élevée $(1,8 \%)$ que

Tableau 1: Croissance annuelle moyenne des cas par tête et dépenses par tête, 2012-2015.

\begin{tabular}{|c|c|c|}
\hline & $\begin{array}{l}\text { Cantons avec des budgets globaux et/ou } \\
\text { des instruments alternatifs de gestion des } \\
\text { quantités durant la période } 2012-2015\end{array}$ & $\begin{array}{l}\text { Cantons sans budgets globaux ni instru- } \\
\text { ments alternatifs de gestion des quantités } \\
\text { durant la période 2012-2015 }\end{array}$ \\
\hline Cas AOS hospitaliers stationnaires (soins somatiques aigus) & $0,6 \%$ & $1,2 \%$ \\
\hline Dépenses AOS hospitalières stationnaires (soins somatiques aigus) & $0,1 \%$ & $0,1 \%$ \\
\hline $\begin{array}{l}\text { Dépenses AOS ambulatoires (secteur ambulatoire des hôpitaux } \\
\text { et des cabinets privés) }\end{array}$ & $4,4 \%$ & $4,7 \%$ \\
\hline Dépenses AOS totales (tous les types de prestations) & $2,7 \%$ & $2,7 \%$ \\
\hline $\begin{array}{l}\text { Dépenses hospitalières stationnaires des cantons (y compris } \\
\text { les PIG*, les investissements cantonaux et la totalité du secteur } \\
\text { hospitalier stationnaire) }\end{array}$ & $1,8 \%$ & $-1,2 \%$ \\
\hline $\begin{array}{l}\text { Dépenses des cantons et AOS hospitalières stationnaires (tota- } \\
\text { lité du secteur hospitalier stationnaire) }\end{array}$ & $1,4 \%$ & $-0,4 \%$ \\
\hline $\begin{array}{l}\text { Dépenses de tous les agents de financement }{ }^{* *} \text { pour le secteur } \\
\text { hospitalier (stationnaire et ambulatoire) }\end{array}$ & $3,2 \%$ & $2,4 \%$ \\
\hline
\end{tabular}
hospitalier (stationnaire et ambulatoire)

* Prestations d'intérêt général; ** Agents de financement: cantons, AOS, autres assurances sociales, ménages privés, assurances complémentaires, autres agents de financement publics et privés.

Source: Lobsiger/Frey (2019, p. 36). 
les cantons sans budgets globaux (-1,2\%). Les changements dans les dépenses cantonales pour les PIG peuvent donc avoir influencé le résultat [22]. De plus, on constate que la réduction des coûts dans les cantons sans budgets globaux est associée à une plus forte diminution des investissements cantonaux dans le contexte du nouveau financement hospitalier [23].

\section{Effets indésirables des budgets globaux cantonaux}

Pour ce qui concerne l'atteinte de l'objectif principal, c'est-à-dire la maîtrise de la croissance des coûts dans le secteur hospitalier, le groupe des cantons avec budgets globaux n'a pas été meilleur que celui des cantons sans budgets globaux. Il faut toutefois s'attendre à des effets indésirables. Parmi les effets indésirables des budgets globaux figurent notamment le rationnement en cas d'épuisement du budget global, la sélection des patients et les temps d'attente pour les interventions électives. Pour évaluer si, dans les cantons avec budgets globaux, les habitants ont davantage eu recours à des prestations extracantonales, les auteurs se sont appuyés sur une étude relative aux flux de patients intercantonaux [24]. Cela a été le cas dans le canton de Neuchâtel durant la période d'analyse (2010-2015), mais pas dans les cantons du Tessin, de Vaud et de Genève. Dans le canton du Tessin, cela peut au moins partielle-

\section{Le développement du système SwissDRG et l'amélioration de la qualité de l'indication sont préférables aux budgets globaux.}

ment s'expliquer par la situation géographique, et dans les cantons de Genève et Vaud, par les hôpitaux universitaires avec leurs larges éventails de prestations [25]. Lors de l'enquête relative à la sélection des patients dans les cantons du Tessin, de Vaud et de Genève, ceuxci ont indiqué ne pas disposer d'indices correspondants [26]. L'existence d'éventuelles listes d'attente n'a pas été analysée plus en détail. Il faut cependant souligner que cette étude et le mandat correspondant ne portaient pas sur ces thèmes.

Pour la FMH, il est donc important d'analyser dans une étude séparée si et dans quelle mesure le rationnement et la sélection des patients sont pratiqués dans les cantons avec budgets globaux, ainsi que l'évolution des temps d'attente pour les patients [27]. Ce faisant, il sera important de ne pas seulement interroger les représentants des cantons, mais aussi les patients et les fournisseurs de prestations (notamment les médecins référents qui sont les plus enclins à fournir une appréciation neutre). Comme les budgets globaux canto- naux existants n'ont pas permis de freiner la croissance des coûts, on peut également se demander dans quelle mesure des budgets globaux plus rigides ou restrictifs accentueront les évolutions défavorables. Finalement, il s'agira aussi d'analyser la charge administrative supplémentaire engendrée par la gestion des budgets globaux.

\section{Faire avancer les alternatives}

Pour les auteurs de l'étude mandatée, les budgets globaux promettent, d'un point de vue théorique, d'atténuer l'incitation à l'accroissement du volume des prestations dû au système des forfaits par cas SwissDRG [28]. Il existe cependant une incitation à l'accroissement du volume notamment pour les prestations qui sont surrémunérées. Dans le système SwissDRG, d'autres prestations sont par contre insuffisamment rémunérées. Depuis des années, la FMH s'engage conjointement avec ses sociétés de discipline pour un système DRG approprié, c'est-à-dire que les prestations ne doivent être ni sur-, ni sous-rémunérées. On citera à ce propos l'engagement dans le cadre de la procédure de demandes de développement du système SwissDRG ou les prises de position sur de nouveaux systèmes SwissDRG et les propositions adressées à SwissDRG SA pour leur développement. SwissDRG SA s'efforce d'ailleurs d'améliorer continuellement le système SwissDRG. De plus, il s'agit de renoncer aux bonus dans les contrats des médecins hospitaliers, en particulier lorsque ceuxci se réfèrent à des objectifs quantifiés [29].

De surcroît, le développement de la mesure de la qualité est essentiel et permet de corriger les éventuelles fausses incitations des DRG. Du point de vue de la FMH, la qualité du diagnostic et de l'indication est un facteur important dans ce contexte. L'ANQ coordonne et réalise la mesure de la qualité sur mandat de SwissDRG SA. Les résultats transparents permettent de déduire des mesures ciblées pour le développement de la qualité dans les hôpitaux et cliniques. La FMH poursuit son engagement également dans ce domaine [30].

\section{Pas d'expériences inutiles avec des mesures inefficaces}

Un objectif important de la politique, celui de freiner la croissance des coûts dans le secteur hospitalier, n'a pas pu être atteint avec les budgets globaux cantonaux. En même temps, les cantons de Genève, Neuchâtel et Vaud paient des PIG supérieures à la moyenne [31]. Si ces cantons veulent réduire les coûts pour les soins stationnaires, pourquoi ne réduisent-ils pas leurs PIG (qui faussent la concurrence) et renoncent en contrepartie à 
un budget global? Et pourquoi les cantons qui appliquent un budget global ont-ils des primes supèrieures à la moyenne [32] et des coûts AOS bruts par tête supérieurs à la moyenne [33]? Compte tenu de ces questions sans réponse, des effets indésirables qui n'ont pas encore été analysés en détail et des alternatives au budget global disponibles, on peut espérer que l'utilisation de cet instrument problématique ne fera pas d'émules.

\section{Références}

1 Objet 14.3385 «Rémunérations forfaitaires et budget global. Evaluation des systèmes en vigueur dans les cantons» (postulat déposé au Conseil national par la Commission de la sécurité sociale et de la santé publique).

2 Communiqué «Le Conseil fédéral examine un objectif de maîtrise des coûts de la santé» (www.admin.ch $\rightarrow$ Documentation $\rightarrow$ Com muniqués).

3 Groupe d'experts sur la maîtrise des coûts (2017). Mesures visant à freiner la hausse des coûts dans l'assurance obligatoire des soins, Berne. (www.bag.admin.ch $\rightarrow$ Assurances $\rightarrow$ Assurance-maladie $\rightarrow$ Maîtrise des coûts).

4 Lobsiger M, Frey M (2019). Budgets globaux dans les cantons dans le domaine des soins stationnaires aigus: application et effets dans le cadre du nouveau financement hospitalier, Basel: B, S, S. Volkswirtschaftliche Beratung (voir www.bag.admin.ch $\rightarrow$ L'OFSP $\rightarrow \mathrm{Pu}-$ blications $\rightarrow$ Rapports d'évaluation $\rightarrow$ Assurance maladie et accidents $\rightarrow$ Révision de la LAMal, financement hospitalier $\rightarrow$ Coûts et financement du système de soins).

5 Lobsiger/Frey (2019, p. 21-23).

6 Lobsiger/Frey (2019, p. 23-29).

7 Lobsiger/Frey (2019, p. 28).

8 Lobsiger/Frey (2019, p. 28 avec référence à l'ATF 138 II 398).

9 Lobsiger/Frey (2019, p. 27).

10 Arrêt du Tribunal administratif fédéral du 16 janvier 2019 (C-5017/2015).

11 Lobsiger/Frey (2019, p. 26 avec référence à l'ATF 138 II 398, E. 4.7).

12 Lobsiger/Frey (2019, p. 26)

13 Déclaration d'Ulrich Kieser lors d'une conférence (voir Kesseli B. Budgets globaux, une fausse bonne idée? Bulletin des médecins suisses 2017;98(47):1562-1565) et dans une interview (voir Balmer H. Globalbudget Krankenversicherung: Patentrezept oder Irrläufer? Clinicum 2018;2:51-55).

14 Felder S (2018). Les budgets globaux: une étape vers les caisses uniques cantonales, p. 8-9 dans: CSS (édit.) Place au dialogue. Quand le système bascule. Le détail des freins aux coûts (voir dialog.css.ch $\rightarrow$ Dossiers $\rightarrow$ Quand le système bascule. Le détail des freins aux coûts).
15 L'étude affecte les cantons qui appliquent d'autres instruments de gestion des quantités ayant un effet comparable aux budgets globaux dans la période d'analyse aux groupes de cantons avec des budgets globaux et parle de "Cantons avec des budgets globaux et/ ou des instruments alternatifs de gestion des quantités" (Lobsiger/Frey, 2019, p. 8-10). Pour simplifier, nous parlons de cantons avec et de cantons sans budgets globaux.

16 AOS: assurance obligatoire des soins.

17 Lobsiger/Frey (2019, p. 36).

18 Lobsiger/Frey (2019, p. 41, 49).

19 Lobsiger/Frey (2019, p. 42).

20 Lobsiger/Frey (2019, p. 49, 51, 66).

21 PIG: prestations d'intérêt général.

22 Lobsiger/Frey (2019, p. 45).

23 Lobsiger/Frey (2019, p. 47).

24 Gruber J, Lipptisch S (2018).Evolution des flux intercantonaux de patients dans le domaine stationnaire (soins somatiques, réadaptation, psychiatrie) entre 2010 et 2015 (seulement en allemand), Zurich: LENZ AG (voir www.bag.admin.ch $\rightarrow$ L'OFSP $\rightarrow$ Publications $\rightarrow$ Rapports d'évaluation $\rightarrow$ Assurance maladie et accidents $\rightarrow$ Révision LAMal, financement hospitalier $\rightarrow$ Paysage hospitalier et garantie de l'approvisionnement en soins)

25 Lobsiger/Frey (2019, p. 59, 85).

26 Lobsiger/Frey (2019, p. 60).

27 Pour la FMH, il serait notamment intéressant d'analyser les délais d'attente pour les traitements à plus long terme découlant du budget global appliqué de fait dans le domaine de la psychiatrie ambulatoire en institutions en Suisse (voir Unger-Köppel J. L'aberration du budget global: le projet-pilote suisse le démontre, Bulletin des médecins suisses, 2017;98(24):753).

28 Lobsiger/Frey (2019, p. 23).

29 Prise de position de la FMH sur les bonus dans les contrats des médecins hospitaliers, novembre 2013.

30 Prise de position de la FMH: Qualité du diagnostic et de l'indication: des dimensions de la qualité sous-estimées, octobre 2012.

31 Voir p. ex. Felder S, Meyer S, Selcik F, Gmünder M (2018). Différences de tarifs et de financement entre hôpitaux publics et cliniques privées dans le secteur des soins aigus stationnaires, 2013-2016. Expertise établie sur mandat de Cliniques Privées Suisses (CPS) (voir www.privatehospitals.ch $\rightarrow$ news); ainsi qu'Ecoplan (2019). Financement des prestations d'intérêt général, des coûts d'utilisation des immobilisations et des garanties de déficit des hôpitaux par les cantons, Berne: Office fédéral de la santé publique (les cantons de Vaud et Neuchâtel n'ont pas fourni de données dans le cadre de cette étude, voir www.bag.admin.ch $\rightarrow$ L'OFSP $\rightarrow$ Publications $\rightarrow$ Rapports d'évaluation $\rightarrow$ Assurance maladie et accidents $\rightarrow$ Révision de la LAMal, financement hospitalier $\rightarrow$ Coûts et financement du système de soins).

32 Voir www.obsan.admin.ch $\rightarrow$ Indicateurs $\rightarrow$ Financement dans le système de santé $\rightarrow$ Primes de l'assurance obligatoire des soins (AOS).

33 Voir www.obsan.admin.ch $\rightarrow$ Indicateurs $\rightarrow$ Financement dans le système de santé $\rightarrow$ Coûts de l'assurance obligatoire des soins (AOS).

\section{\{FMH

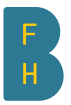

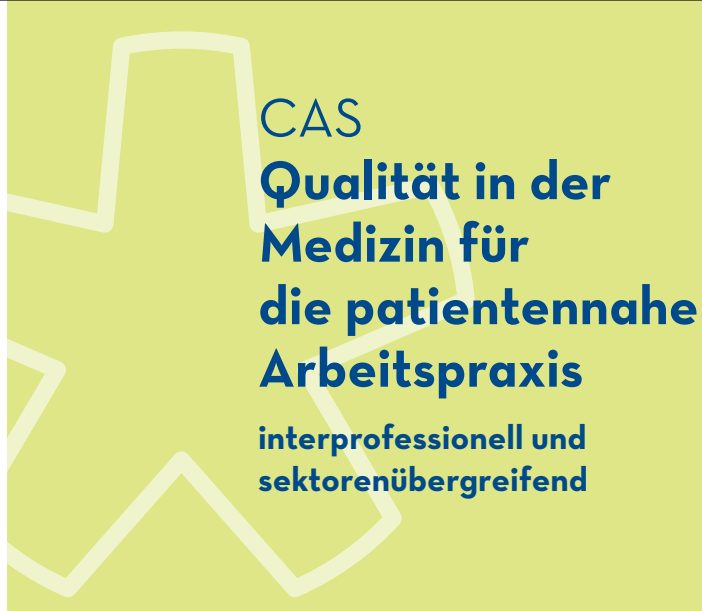

Werden Sie zu Spezialistinnen und Spezialisten im Qualitätsmanagement! Sie lernen, eigenständig Projekte in den Bereichen Qualitätsentwicklung und Patientensicherheit zu erarbeiten und umzusetzen.
Kursdauer

März bis September $2 \mathrm{O} 2 \mathrm{O}$

Anmeldung

bis 3.2.2O2O unter

bfh.ch/gesundheit/

weiterbildung/cas/

qualitaet-in-der-medizin 